North America Regional Plenary IGCS19-0247

\section{VARIATIONS IN ENDOMETRIAL CANCER RISK AND HISTOLOGIC DISTRIBUTION BY CARIBBEAN NATIVITY}

${ }^{1} \mathrm{M}$ Schlumbrecht*, ${ }^{1} \mathrm{~S}$ George, ${ }^{2} \mathrm{~A}$ Pinto, ${ }^{1} \mathrm{M}$ Huang, ${ }^{1} \mathrm{~B}$ Slomovitz, ${ }^{3} \mathrm{~T}$ Koru-Sengul. ${ }^{1}$ Sylvester Comprehensive Cancer Center, Division of Gynecologic Oncology, Miami, USA; 2University of Miami, Department of Pathology, Miami, USA; ${ }^{3}$ University of Miami, Department of Public Health Sciences, Miami, USA

10.1136/ijgc-2019-IGCS.48

Objectives Prior data suggest that within subpopulations of racial and ethnic minorities, endometrial cancer (EC) risk varies. Nativity as mediator of risk has been poorly studied. Our objective was to determine if Caribbean nativity influences EC risk and presentation.

Methods Using the Florida Cancer Data System (FCDS), we identified women diagnosed with EC from 1981-2013. Demographics and pathologic factors were abstracted. Caribbean nativity included countries of both African and Hispanic lineage. Statistical analyses were performed using logistic regression and chi-square, with statistical significance at $\mathrm{p}<0.05$.

Results Of the 23,690 women in the FCDS identified with EC, 840 had Caribbean nativity. Among Caribbean immigrants with EC, a higher proportion had type II histologies compared to US natives $(35.6 \%$ vs. $27.5 \%, \mathrm{p}<0.01)$, with very high prevalence seen among Haitian $(54.2 \%, \mathrm{p}<0.01)$ and Jamaican $(47.1 \%, \mathrm{p}<0.01)$ women. In comparison to US-born women, uterine serous carcinoma was more prevalent in women from Guyana $(22.2 \%$ vs. $6.5 \%, p=0.03)$ and Jamaica $(13.8 \%$ vs. $6.5 \%, \mathrm{p}=0.001)$, with carcinosarcoma more prevalent in women from Haiti $(13.5 \%$ vs. $4.6 \%, \mathrm{p}<0.01)$ and Jamaica $(13.8 \%$ vs. $4.6 \%, \mathrm{p}<0.01)$. More Caribbean immigrants presented with distant disease $(10.1 \%$ vs. $6.9 \%$, $\mathrm{p}<0.01)$. Relative to US natives, significant increases in type II EC risk were seen in women born in Haiti (OR 3.08 [2.064.62], $\mathrm{p}<0.01$ ) and Jamaica (OR 2.32 [1.66-3.25], $\mathrm{p}<0.01$ ).

Conclusions Women of Jamaican and Haitian birthplace have increased risks of type II EC compared to US-born women. Caribbean natives also have a higher prevalence of type II histologies. Effect of nativity on EC warrants further study.

\section{IGCS19-0350}

\section{CIRCULATING CELL-FREE TUMOUR DNA FOR SURVEILLANCE OF ENDOMETRIAL AND OVARIAN CARCINOMA}

${ }^{1} \mathrm{~A}$ Lum, 'S Lam, 'M Nazeran, 'W Yang, ${ }^{1} \mathrm{~J}$ Senz, ${ }^{2} \mathrm{R}$ Hernandez, ${ }^{2} \mathrm{~S}$ Malikic, ${ }^{2} \mathrm{M}$ McConechy, ${ }^{3}$ DG Huntsman, ${ }^{4} \mathrm{~J}$ McAlpine*. 'University of British Columbia, Pathology, Vancouver, Canada; ${ }^{2}$ Contextual Genomics, Bioinformatics, Vancouver, Canada; ${ }^{3}$ University of British Columbia and BC Cancer Agency, Pathology, Vancouver, Canada; ${ }^{4}$ University of British Columbia and BC Cancer Agency, Gynecology/Gynecologic Oncology, Vancouver, Canada

\subsection{6/ijgc-2019-IGCS.49}

Objectives We sought to determine the feasibility and characterize the extinction kinetics of circulating cell-free tumor DNA (cfDNA) testing in endometrial and ovarian carcinomas (ECs, OCs) using a clinically-approved commercially-available assay.
Methods Women with suspected EC/OC undergoing surgery were consented for tissue and plasma sampling including pre-operative and serial post-operative draws. Tumour tissue and patient-matched buffy coat was extracted for DNA and sequenced for somatic mutations using FINDIT $^{\mathrm{TM}}$ panel assay. Plasma samples were extracted for cfDNA and sequenced using FOLLOWIT ${ }^{\mathrm{TM}}$, Illumina platform, and analyzed using Contextual Genomics's QUALITY NEXUS analysis pipelines. Low-frequency variants were confirmed by digital droplet PCR.

Results 44 individuals had sufficient tissue and follow-up for inclusion; 24 ECs (13 endometrioid, 10 high-grade serous (HGS), 1 clear cell(CC)), 18 OCs (17 HGS 1, CC), and 2 synchronous endometrial and ovarian carcinomas. Eight ECs and 15 OC cases were advanced stage (II-IV) with residual disease in 2 ECs and 5 OCs, 8 recurrence events and 3 deaths recorded. Compliance with plasma sampling was $\operatorname{high}(>95 \%)$ when requested in hospital or at routine surveillance visits but dropped to $68 \%$ for 'extra' study-associated visits. Analysis to date reveals cfDNA was detectable in pre-operative samples of 19 individuals (9 ECs, 10 OCs including 4 early stage) and 6/10 tested post-operatively. Normalization of conventional tumour markers post-operatively took a median of $3 \mathrm{mo}$ in contrast to rapid loss of detectable cfDNA.

Conclusions cfDNA testing is feasible and may enhance surveillance of endometrial and ovarian carcinomas by reflecting i) volume of disease pre-/post-operatively, ii) response to therapy, and/or iii) recurrence.

\section{IGCS19-0481}

\section{AGE, HISTOLOGY AND STAGE PREDICT SURVIVAL FOLLOWING ADJUVANT CHEMOTHERAPY AND RADIATION VERSUS RADIATION ALONE IN HIGH-RISK ENDOMETRIAL CANCER: A STUDY BASED ON PORTEC-3 CRITERIA}

$1,2 \mathrm{Y}$ Casablanca* ${ }^{1,3} \mathrm{C}$ Tian ${ }^{4} \mathrm{M}$ Powell ${ }^{5} \mathrm{~B}$ Winterhoff, ${ }^{6} \mathrm{JK}$ Chan ${ }^{2} \mathrm{CD}$ Shriver, ${ }^{7} \mathrm{CA}$ Hamilton, ${ }^{7} \mathrm{GL}$ Maxwell, 1,2,3 KM Darcy. ${ }^{1}$ Uniformed Services University of the Health Sciences, Gynecologic Cancer Center of Excellence, Bethesda, USA; ${ }^{2}$ Uniformed Services University of the Health Sciences, John P Murtha Cancer Center Research Program, Bethesda, USA; ${ }^{3}$ Henry Jackson Foundation for the Advancement of Military Medicine- Inc., Women's Health Integrated Research Center, Annandale, USA; ${ }^{4}$ Washington University St Louis, Gynecologic Oncology, St Louis, USA; ${ }^{5}$ University of Minnesota, Gynecologic Oncology, Minneapolis, USA; ${ }^{6}$ Palo Alto Medical Foundation- California Pacific Medical Center- Sutter Health, Gynecologic Oncology, San Francisco, USA; ' Inova Fairfax Hospital and Inova Shar Cancer Institute, Gynecologic Oncology, Falls Church, USA

\subsection{6/ijgc-2019-IGCS.50}

Objectives Evaluate the impact of age, stage and histology on survival in high-risk endometrial cancer (EC) following treatment with chemotherapy and radiation (CTRT) vs. radiation alone (RT).

Methods Eligible patients were diagnosed with high-risk EC from 2004-2014 in the National Cancer Database based on PORTEC-3 criteria and treated with pelvic beam radiation and/or radioactive implants. The CTRT group also received multiple-agent chemotherapy. A propensity score approach controlled for differences in clinical factors. Survival was evaluated using weighted Kaplan-Meier and Cox model analyses with interaction testing. 